\section{TWO UNUSUAL CASES OF ABDOMINAL TUBERCULOSIS.}

\author{
BY
}

E. G. SLESINGER, O.B.E., M.S., F.R.C.S. ASSISTANT SURGEON, GUY'S HOSPITAL.

THE two following cases appear to be sufficiently unusual to be worth recording, partly on account of the difficulties in diagnosis and partly on account of the apparent rarity of mesenteric abscess at the ages at which it occurred in these two patients.

\section{CASE I.}

H. P., a male infant aged 5 months, had been noticed to appear ill for ten days. Diarrhoea was present during the whole of this period, the stools being greenish and offensive, but not containing any blood. The child had been taking its food badly and had vomited four times before it was brought to the hospital, when it was at once admitted.

On admission he looked extremely ill. He was pale, with sunken face and restless. The abdomen was very greatly distended, appeared to be tender, was resonant in the middle and dull in the flanks. The child vomited once in the ward, but the vomit was in no way characteristic. Pulse very feeblo 150 to 160 , temperature $102^{\circ}$. The abdomen was too tensely distended for anything to be palpated, and the most probable diagnosis seemed to be a pneumococcal peritonitis.

Laparotomy was performed under a spinal anaesthetic. On opening the abdomen a large quantity of purulent tluid and intening a large quantity of purulent thuid and intestinal contents escaped, and a loop of intestine, showing perforation, presented. The bowel wall in the neighbourhoo and over most of the coil was oedematous and obviousl ulcerated. The perforation was closed and was reinforced with an omental graft owing to the difficulty of making the sutures hold satisfactorily. It was noticed that the greater part of the pus came from a mass which could be felt in the abdomen, and this on further exploration proved to be a mesenteric abscess. from which several ounces of pus were evacuated. A tube was left draining the abscess cavity and Carrel instillation of the peritoneal cavity with saline was instituted. The child appeared to rally at first, but died the following morning.

Post-mortem examination showed extensive tuberculous ulcera tion of the small intestine; one of the ulcers had perforated and the cavity of the mesenteric abscess was in relation to ruptured tuberculous caseating gland.

CASE II.

F. J., a male aged 34, gave a history of pain in the left ilia fossa for three weeks, the pain being also referred to both lumbar resions. 'It was not influenced by food or exercise, bu was a good deal worse at night. The patient had been extremely constipated, but had never vomited. He was admitted to hospital as a case of subacute intestinal obstruction. There wa some abdominal distension, with tendernegs over the left iliac fossa; a tumour of somewhat vague contour, but of firm sistence, could be palpated in this region. manipulation was painful. The tongue was furred and dry and an pulation was to act. Tempera $994^{\circ}$ pulse 100 . Th , and an enema failed abscess walled in by bowel, but as auscultation and palpation revealed the caecum in the normal position and apparently not revealed the caecum in the normal position and apparently not connected with the tumour, a tentative diagnosis of an abscess culitis was considered, but seemed unlikely on account of the

Laparotomy was performed, and the tumour explored. The appendix was attaclied by its tip to the tumour, and was secondarily somewhat infiamed, and consequently removed. A well formed Meckel's diverticulum was separated from the loops of bowel bounding the mass, but was geen to be normal apart from some injection. The mesentery of the small intestine was noted to be very thick and short, the bowel being bound down almost to the posterior abdominal wall; on separating the loops, about a pint of faecal-smelling pus was evacuated. On exploring the cavity, it was found to be bounded by mesentery and bowel, and from one wall half of a semi-calcareous gland was removed. In spite of the faeculent odour of the pus, gland was removed. In spite of the faeculent odour of the pus, was drained, and the patient made an uninterrupted recovery, was drained, and the patient made an
the track of the drain healing quickly.

The unusual points about the first case were the age of the patient-perforation of a tuberculous ulcer in infancy must be very uncommon-and the presence of a solitary well-marked abscess originating in a tuberculous mesenteric gland. The condition of the child was such that death appeared inevitable, but it was felt that operation offered the only chance.

There appears no doubt that in the second case the mesenteric abscess originated in an old tuberculous gland, and a few calcareous glands were felt elsewhere in the mesentery. The signs and symptoms were exactly those of an appendicular abscess occurring on the left side, but the caecum could be easily felt on the rigit. The tumour was comparatively freely movable in all directions, which was unlike diverticulitis; it felt like a walled-in abscess of some lind. There was no history suggesting tabes mesenterica during oliildhood, nor was there anything to account for the sudden flaring up of a tuberculous gland which must have been present for some time. 'The faecu. lent odour was probably due to the commencing peritonitis on the adjacent coils of intestine; in view of the fact that cultures remained sterile, it is difficult to account for the development of the abscess by organisms passing from the bowel along the lymphatics, which at first sight appeared the most probable explanation.

\section{Ettentaranda:}

\section{MEDICAL, SURGICAL, OBSTETRICAL.}

\section{PAIN IN PAROXYSMAL AURICULAR FIBRILLATION.}

PaIN is not a common accompaniment of auricular fibril. lation (Mackenzie), and the following case therefore seems of sufficient interest to be recorded:

A man, aged 52, had a "cold" at the end of October, 1916. On November 3 rd, on his way to business, he noticed that hedid not walk quite so easily as usual owing to tightness in the chest, and when he got on the top of a tram he felt fluttering in chest, and when he got on the top of a tram he felt fluttering in
his chest. This continned, but he managed to do his work. Next day he had pain in the left chest, which made it difficult Next day he had pain in the left chest, which made it difficult
for him to take a long breath. When seen on November 5 th lie for him to take a long breath. When seen on November 5 th he lay in bed, breathing easily, but complaining of fluttering and a severe pain over a small area of the front of the left chest at
the level of the third rib near the sternum. Here there was a the level of the third rib near the sternum. Here there was a
patch of skin, about 1 in. in diameter, hypersensitive to pinprick and light pinching. The pulse rate was rather rapid (95 to 100) and grossly irregular. 'The fluttering was chiefly felt at the same place as the pain. No dilatation of the lieart could be demonstrated ; there were no murmurs and no friction sound. A polygraph curve showed ventricular venous pulse and absolutely irregular rhythm (auricular fibrillatios). The pain continued during the following days, though less severe. On November 14th the pulse was found to be regular, at 56 per minute. The previous evening he had felt rapid relief from pain, and found that the irregularity of the pulse had disappeared. A polygraph curve now showed the normal rhythm.

'I'wo years later, after a slight illness, he had another attack of fibrillation, induced apparently by running for a tram, and the pulse has remained irregular to the present time. Fortunately for the patient the heart rate is moderate, ranging about 70, and by planning out his work so as to avoid hurry he is able to carry on an active life.

The pain in this case did not appear immediately after the onset of fibrillation, but only when, the patient having endeavoured to carry on his work for a day, an additional strain was pat on an embarrassed circulation. Rest in bed lessened the pain, but it did not cease until the normal rhythm was restored, and then its disappearance was rapid and complete. Hull.

E. E. Laslett, M.D.

\section{GLANDULAR FEVER.}

I HAvE recently observed in my practice, which lies in the extreme north of Scotland, a number of cases of "sore throat," usually slight, accompanied by cervical glandular enlargements quite out of proportion to the condition of the fances and pharynx. I may say that a few of those cases showed a striking resemblance to cases described as glandular ferer.

The glands involved were those situated beneath the sterno-mastoid muscle, about the upper part of its middle third and below the level of the angle of the jaw. Lack of symmetry of the two sides was also a feature. 'I'he skin over the glands was not reddened or oedematous. The glands remained discrete and showed no tendency to suppurate, and some of them were as large as a pigeon's egg, and even larger. As already mentioned, the throat condition was slight, and in at least one case the throat appeared to be quite normal.

The history of the most recent case I observed is briefly this :

A girl, aged 4 rears, complained of being nnwell one evening, and the mother noticed a swelling on the left side of the neck. There were no other children in the house. When I saw her next morning the child presented the following clinical picture: Pyrexia with rapid pulse, vomiting and furred tongue, great Pyrexia with rapid pulse, vomiting and furred tongue, great of the angle of the jaw, situated roughly under tre middle third of the angle of the jaw, situated roughly under tre middle third
of the sterno-mastoid muscle; no glands were palpable on the 